This work was in part supported by a grant from FOMRE. We would like to thank the Reactor Centrum Nederland (RCN), Petten, for their kind hospitality. We are greatly indebted to $\mathrm{Dr} \mathrm{H}$. M. Rietveld for his help and generous provision of neutrons.

\section{References}

Bacon, G. E. (1955). Neutron Diffraction. Oxford Univ. Press.

Batsanov, S. S. \& Gorogotskaya, C. I. (1959). Izvest. Vysshik Ucheb Zavadeniil, Khim. i. Khim. Tekhnol, 2, 858.

Benjamin, E. \& Westrum, E. F. Jr (1957). J. Amer. Chem. Soc. 79, 287.

Busing, W. R., Martin, K. O. \& Levy, H. A. (1962). ORFLS. Oak Ridge National Laboratory, Oak Ridge, Tennessee.

Cromer, D. T., Kay, M. I. \& Larson, A. C. (1967). Acta Cryst. 22, 182.

GMELIN, (1936). Gmelins Handbuch, 23, 145.

Groenewegen, P. P. M. \& FeIL, D. (1969). Acta Cryst. A 25, 444.

Hamiton, W. C. (1957). Acta Cryst. 10, 629.

Hamilton, W. C. (1965). Acta Cryst. 18, 502.
Hansen, H. P., Herman, F., Lea, J. D. \& Skillman, S. (1964). Acta Cryst. 17, 1040.

Ibers, J. A. \& Stevenson, D. P. (1958). J. Chem. Phys. 28,929. International Tables for X-ray Crystallography (1962). Vol. III, p. 202. Birmingham: Kynoch Press.

Jeffrey, G. A., Parry, G. S. \& Mozzi, R. L. (1956). J. Chem. Phys. 25, 1024.

KamiYoshi, K. (1956). Sci. Rep. Res. Insts. Tòhoko Univ., Ser. A 8, 252.

Keffer, F. \& Portis, A. M. (1957). J. Chem. Phys. 21, 675.

Morosin, B. \& Shirber, J. E. (1965). J. Chem. Phys, 42, 1389.

Peterson, S. W. \& Levy, H. A. (1957). Acta Cryst. 10, 70.

Plumb, R. C. \& Hornig, D. F. (1955). J. Chem. Phys. 27, 947.

Rush, J. J., Taylor, T. I. \& Havens, W. W. JR (1960). Phys. Rev. Letters, 5, 507.

Shulvas-Sorokina, R. D. \& Evdomikov, V. G. (1939). Acta Physicochim. U.S.S.R. 11, 291.

Simon, F., Simpson, v. C. \& RuhemanN, M. (1927). $Z$. Phys. Chem. 129, 345.

Stevenson, R. (1960). J. Chem. Phys. 34, 346.

Stewart, R. F. Davidson, E. R. \& Simpson, W. T. (1963). J. Chem. Phys. 42, 3175.

Zachariasen, W. (1927). Z. Phys. Chem. 127, 218.

Acta Cryst. (1969). A25, 444

\title{
Molecular Form Factors in X-ray Crystallography
}

\author{
By P.P. M. GroeneweGen AND D. FeIL \\ Department of Chemical Technology, Technische Hogeschool Twente, Enschede, The Netherlands
}

(Received 25 September 1968)

\begin{abstract}
The calculation of molecular form factors from ab initio molecular electronic wave functions is discussed, and a scheme for application to X-ray diffraction structure analysis is given. The method is used to calculate the form factor of the $\mathrm{NH}_{4}^{+}$molecular ion from three accurate molecular wave functions, as well as for the corresponding isolated-atoms arrangement. The merits of the different form factors obtained are tested on experimental single-crystal X-ray diffraction data for $\mathrm{NH}_{4} \mathrm{~F}$. It appears that the quality of the form factor is determined by the number of centres in the wave function basis rather than by the wave function energy.
\end{abstract}

\section{Introduction}

In X-ray diffraction structure analysis the electron density distribution in crystalline solids is usually resolved in terms of distinct, i.e. isolated, atoms or ions. Interatomic effects of chemical and general solid state binding are obscured in this scheme and resist quantitative evaluation. Efforts to account $a$ priori for the effect of binding have been directed mainly at evaluating scattering factors for 'bonded' and 'valence state' atoms (McWeeny, 1951, 1952, 1953, 1954; Freeman, 1959; Dawson, 1964; Stewart, Davidson \& Simpson, 1965). The use of molecular form factors calculated from $a b$ initio molecular wave func- tions is feasible for structures that may be resolved in terms of small molecules or molecular ions. Application of this method to X-ray crystallography seems to be non-existent or of questionable significance (Webb, 1965; amended by Davis \& Whitaker, 1966). Some success has been had with the use of a molecular form factor in the interpretation of electron scattering data from $\mathrm{CH}_{4}$ (Iijima, Bonham, Tavard \& Roux, 1965).

In this work we discuss the calculation of molecular form factors from $a b$ initio wave functions and their application to X-ray diffraction structure analysis. The method is demonstrated for the $\mathrm{NH}_{4}^{+}$molecular ion in the $\mathrm{NH}_{4} \mathrm{~F}$ structure. Results of subsequent few parameter structure refinements are given. 


\section{Calculation of molecular form factors}

The form factor (or coherent X-ray scattering factor) for an $N$-electron system (a molecule) is, within the first Born approximation,

$$
f(\mathbf{s})=\left\langle\Psi\left|\sum_{i=1}^{N} \exp \left(i \mathbf{s} . \mathbf{r}_{i}\right)\right| \Psi\right\rangle,
$$

where $\mathbf{s}$ is equal to $\mathbf{k}-\mathbf{k}_{0}$, where $\mathbf{k}_{0}$ and $\mathbf{k}$ are the wave vectors of the incident and scattered beam, and $\mathbf{r}_{i}$ denotes the position coordinate of the $i$ th electron with respect to a common origin. For $\Psi$ we take the ground state electronic wave function in a fixed-nuclei configuration. If $\Psi$ is exact, or a properly antisymmetrized approximation, $f(\mathbf{s})$ reduces to the Fourier transform of the one-electron probability distribution function:

$$
\begin{aligned}
& f(\mathbf{s})=N \int d \mathbf{r} \varrho(\mathbf{r}) \exp (i \mathbf{s} . \mathbf{r}), \\
& \varrho(\mathbf{r})=\left\langle\Psi\left|\delta\left(\mathbf{r}-\mathbf{r}_{i}\right)\right| \Psi\right\rangle \\
& \text { (H. F.) } \\
& =N^{-1} \sum_{i=1}^{N}\left|\varphi_{i}(\mathbf{r})\right|^{2},
\end{aligned}
$$

where, in the second line of equation (3) we have restricted ourselves to approximate wave functions of Hartree-Fock form, since inclusion of configuration interaction terms adds no new features to the nature of the calculations. The one-electron orbitals $\varphi_{i}(\mathbf{r})$ (space coordinate functions) are taken to be constructed by linear combination of certain basis functions $\chi_{p}(\mathbf{r})$ :

$$
\varphi_{i}(\mathbf{r})=\sum_{\mathbf{p}} C_{i p} \chi_{p}(\mathbf{r}) .
$$

In terms of these basis functions the form factor of the system becomes:

$$
\begin{gathered}
f(\mathbf{s})=\sum_{i=1}^{N} \sum_{p, q} C_{i p} C_{i q} f_{p q}(\mathbf{s}), \\
f_{p q}(\mathbf{s})=\int d \mathbf{r} \chi_{p}(\mathbf{r}) \chi_{q}(\mathbf{r}) \exp (i \mathbf{s} . \mathbf{r}),
\end{gathered}
$$

where for convenience the linear coefficients and basis functions have been assumed to be real. The problem of calculating the elements $f_{p q}(\mathbf{s})$ contributing to $f(\mathbf{s})$ is now determined by the nature of the basis $\left\{\chi_{p}\right\}$.

The basis functions $\chi_{p}$ are usually of factorized form with respect to some origin (atomic nucleus),

$$
\chi_{p}\left(\mathbf{r}^{\prime}\right)=\varrho_{p}\left(\mathbf{r}^{\prime}\right) S_{l m}\left(\theta^{\prime}, \varphi^{\prime}\right),
$$

where $S_{l m}$ is a real surface harmonic and the primes indicate that the coordinates refer to the origin of factorization. In the following there is little opportunity for confusing the one-electron probability distribution, $\varrho$, with the radial functions $\varrho_{p}$. Since more centres may be present in the basis $\left\{\chi_{p}\right\}$ (in the case of multi-centre wave functions) the integrals $f_{p q}(\mathbf{s})$ may be of one (O) centre (C) or of two centre (TC) type. As shown in the Appendix, OC integrals can be reduced to derivatives of simple analytic basic forms if the radial basis functions, $\varrho_{p}$, are analytic (of Slater or Gaussian type), while for numerical $\varrho_{p}$ one-dimensional integration has to be performed numerically. TC integrals can be reduced to derivatives of basic forms if the radial basis is Gaussian, while for a Slater radial basis, projection of the $\varrho_{p}$ on a Gaussian basis has been demonstrated by McWeeny (1953) for a special case.

Within the same formalism the wave function for an isolated-atoms arrangement is an antisymmetrized product of single-atom wave functions. Here we have a multi-centre basis $\left\{\chi_{p}\right\}$; no TC elements $f_{p q}(\mathbf{s})$ arise, however, as any orbital is constructed from commoncentre basis functions only.

The resulting molecular form factor $f(\mathbf{s})$ constitutes, in general, a three-dimensional field of numbers, within which the symmetry of the molecule is preserved. Tabulation of $f(\mathbf{s})$ is, therefore, not attractive. Instead, for profitable application in X-ray diffraction structure analysis, required values of $f$ for measured reflexions $\mathbf{s}(h, k, l)$ are calculated directly from the available wave function.

\section{Application in X-ray crystallography}

The present approach resolves the electron distribution in crystalline solids in terms of distinct molecules, molecular ions, atoms and/or ions. In the following all these systems are regarded as molecules. Therefore, the summation in the familiar expression for the structure factor $F$ for a particular reflexion $\mathbf{s}$,

$$
F(\mathbf{s})=\sum_{j} f_{j}(\mathbf{s}) T_{j}(\mathbf{s}) \exp \left(i \mathbf{s} . \mathbf{R}_{j}\right),
$$

is now over all distinct molecules in the crystallographic unit cell. In (8), $T_{j}=\exp \left(-\mathbf{s} . \mathbf{B}_{j} . \mathbf{s}\right)$ and $\mathbf{R}_{j}$ denote the temperature factor and position of molecule $j$.

It is seen that in this formalism each distinct system has one temperature factor, implying the treatment of molecules as rigid scatterers. The seriousness of this restriction is not easy to estimate, since it is related to the problem of the distribution of lattice vibrational modes contributing to the mean-square displacements of the atoms in the structure. It seems that at sufficiently low temperatures the high frequency contributions become less important (James, 1948), justifying to some extent the use of small rigid scatterers.

Now the vector $\mathbf{s}$ in (8) for reflexion $h k l$ is defined with respect to the crystallographic unit-cell coordinate system $(a, b, c)$ while for calculation of $f_{f}(\mathbf{s})$ the (Cartesian) components of $\mathbf{s}$ are needed in the molecular coordinate system $\left(x_{j}, y_{j}, z_{j}\right)$ of molecule $j$ (as determined for convenience of wave function calculation reflected by the choice of the basis $\left.\left\{\chi_{p}\right\}\right)$. 
Hence, transformation matrices $\mathbf{K}_{j}$ are required to transform $\mathbf{s}(a, b, c)$ to $\mathbf{s}\left(x_{j}, y_{j}, z_{j}\right)$ for each molecule $j$ (different $\mathbf{K}_{\boldsymbol{j}}$ may, of course, be related by the symmetry properties of the structure). Therefore, in order to apply molecular form factors in structure analysis, a preliminary analysis in terms of distinct atoms must be carried out to establish the $\mathbf{R}_{j}$ and $\mathbf{K}_{j}$.

The overall procedure of structure analysis in terms of molecules is pictured in Fig. 1. The experiment defines the set of observed reflexions, indicated by $\{\mathbf{s}(a, b, c)\}$. A conventional structure analysis (1) in terms of atoms provides the positons of all atoms in the unit cell and hence defines the set, $\left[\mathbf{R}_{j}, \mathbf{K}_{j}\right]$, of positions and orientations of all distinct molecules with respect to the unit-cell coordinate system. Application of the $\mathbf{K}_{j}$ to set $\{\mathbf{s}(a, b, c)\}$ produces the sets $\left\{\mathbf{s}\left(x_{j}, y_{j}, z_{j}\right)\right\}$.
In the next stage the sets $\left\{f_{j}\right\}$ are calculated from the available wave function(s) as discussed in $\S 1$. These then allow for calculation of $\left\{F_{\text {calc }}\right\}$ which is compared with $\left\{I_{\mathrm{obs}}\right\}$ in a final structure analysis (2) in terms of molecules, in order to refine remaining parameters and establish agreement indices.

An overall feature of the procedure is a reduction of the number of adjustable parameters, since in analysis (2) only positions $\mathbf{R}_{j}$ and temperature factors $T_{j}$ of molecules may be refined. (Orientations $\mathbf{K}_{j}$ of molecules may be varied if one is prepared to go back in the procedure and recalculate $\left[\left\{f_{j}\right\}\right]$.) An interesting possibility, not explored in the present investigation, is the variation of nuclear positions in the wave function and a study of the resultant effect on crystallographic analysis.

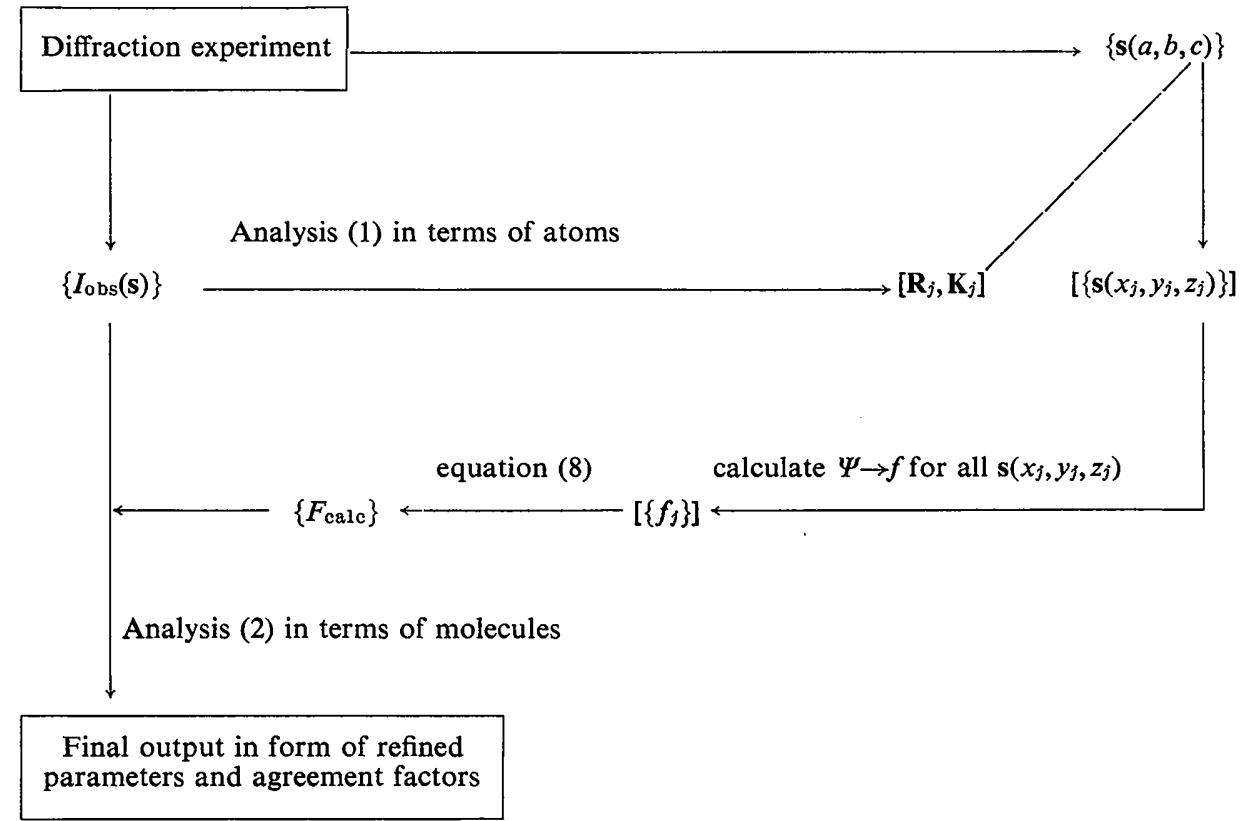

Fig. 1. Procedure for structure analysis in terms of distinct molecules. Curly brackets denote the set of observed reflexions. Square brackets denote the set of molecules in the crystallographic unit cell.

Table 1. Specification of $\mathrm{NH}_{4}^{+}$wave functions; calculation of corresponding form factors

Reference
Approximation ${ }^{a}$
Energy (a.u.)
Type radial basis ${ }^{b}$
Dimension radial basis
Number lin. coeff.
N-H distance (a.u.)
f calculation procedure
Computing time $^{e}$

$\begin{array}{cc}\Psi_{\mathrm{K}} & \Psi_{\mathrm{M}} \\ \text { Krauss } & \text { Moccia } \\ (1963) & (1964) \\ \text { HF } & \text { HF } \\ -56 \cdot 5038 & -56 \cdot 306 \\ \text { MC-G } & \text { OC-S } \\ 16 & 11 \\ 31 & 18 \\ 1.9464 & 1.9900^{c} \\ \text { A } & \text { A } \\ 27 & 9\end{array}$

$\Psi_{\mathrm{AC}}$
Albasiny-Cooper
$(1963)$
$\mathrm{HF}$
$-56 \cdot 306$
$\mathrm{OC}-\mathrm{N}$
6
$1 \cdot 90^{c}$
$\mathrm{~N}$
55

$\left[\Pi \Psi_{n}\right]$

Clementi

(1965)

IA

$-55 \cdot 690$

MC-S

9
14

optional

$f$ calculation proced
Computing time

9

A
(a) Hartree-Fock; isolated atoms.
(b) Multi-centre or one-centre; Gaussian, Slater or numerical.
(c) Near calculated equilibrium distance.
(d) Analytical or by numerical integration.
(e) In seconds, for one value of $\mathbf{s}$. 


\section{Form factor of $\mathrm{NH}_{4}^{+}$}

The form factor of the $\mathrm{NH}_{4}^{+}$molecular ion was calculated from three accurate (judged by the calculated energy) molecular wave functions (Krauss, 1963; Moccia, 1964; Albasiny \& Cooper, 1963), and for the corresponding isolated-atoms arrangement. Information on the different wave functions is collected in Table 1. The Albasiny-Cooper wave function results from numerical solution of the Hartree-Fock equations and the radial functions obtained are available tabulated at 57 argument values.*

In the last column of Table 1 an isolated-atoms wave function is given, an antisymmetrized product of one $\mathrm{N}^{+}$and four properly centered $\mathrm{H}$ atomic wave functions. For the open shell $\mathrm{N}^{+}$ion a ${ }^{1} S$ wave function (Clementi, 1965) was chosen, since the other (lower energy) states have aspherical one-electron density distributions which are not invariant under the $T_{d}$ symmetry group of $\mathrm{NH}_{4}^{+}$. This ${ }^{1} S$ wave function is actually of configuration interaction form, but the calculations are completely analogous to the H.F. case. The energy of the isolated-atoms system is the sum of the $\mathrm{N}^{+}$and four (free) $\mathrm{H}$ atomic energies, and is independent of the $\mathrm{N}-\mathrm{H}$ distance.

Form factors were calculated for all wave functions of Table 1 . The average times needed for computing $f_{\mathrm{NH}_{4}^{+}}^{+}$for a particular vector $\mathrm{s}$, listed in Table 1 , refer

* We thank Dr Albasiny for providing us with additional information on the integration procedure to be employed for the radial functions. to an IBM system $360 / 30$ computer. These times may be considered upper limits since improvement of the programs is probable. A set $\{f\}$, corresponding to a number of values $\mathbf{s}$, shows $f$ decreasing with $\mathbf{s}$ in a manner familiar from atomic form factors, with a wealth of detail dependent on the orientation of $\mathbf{s}$ with respect to the molecule superimposed. Since $\{f\}$ corresponds to the Fourier transform of the one electron density distribution of the $\mathrm{NH}_{4}^{+}$ion, effects of chemical bonding will be preserved in $\{f\}$ if the resolution of the set is fine enough.

\section{Application to $\mathrm{NH}_{4} \mathrm{~F}$ structure analysis}

The structure of $\mathrm{NH}_{4} \mathrm{~F}$ has recently been studied in this laboratory with X-ray and neutron diffraction by Adrian (1968) and Adrian \& Feil (1969), from whose work we quote the following results of importance for the present investigation:

The $\mathrm{NH}_{4}^{+}$ion does not rotate at room temperature. The symmetry of the $\mathrm{NH}_{4}^{+}$ion is almost tetrahedral. ( $\mathrm{NH}_{4} \mathrm{~F}$ has the wurtzite structure with an $u$ parameter of 0.378 . This positional parameter determines the relative positions of the two h.c.p. sublattices.)

Thermal motion of the $\mathrm{N}$ and $\mathrm{F}$ nuclei is approximately isotropic (individual isotropic temperature factors from neutron diffraction are $B_{F}=0.82$ and $B_{N}=$ 0.86 at $\left.-196^{\circ} \mathrm{C}\right)$.

The $\mathrm{N}-\mathrm{H} \cdots \mathrm{F}$ hydrogen bond displays little concentration of electronic charge in between $\mathrm{H}$ and $\mathrm{F}$.

Refined $\mathrm{N}-\mathrm{H}$ distances are 1.95 a. u. (neutron diffraction) and 1.75 a.u. (X-ray diffraction).

Table 2. Results of the 4 parameter (overall scale factor, individual isotropic temperature factors, one positional parameter) refinement $\mathrm{NH}_{4} \mathrm{~F}$ crystal structure at $-155^{\circ} \mathrm{C}$

\begin{tabular}{|c|c|c|c|c|c|c|}
\hline \multirow{2}{*}{$\begin{array}{l}\text { Wave function } \\
\text { employed for } \mathrm{NH}_{4}+\end{array}$} & \multicolumn{4}{|c|}{ Refined parameters } & \multicolumn{2}{|c|}{ Agreement } \\
\hline & o.s.f. & $B_{F}$ & $B_{N}$ & $u$ & $R$ & $R_{w}$ \\
\hline Krauss (1963) & $16 \cdot 53(14)^{*}$ & $1 \cdot 10(2)$ & $1.06(2)$ & $0.3778(2)$ & $1.8 \dagger$ & $2 \cdot 6 \dagger$ \\
\hline Moccia (1964) & $17 \cdot 04(40)$ & $1 \cdot 14(6)$ & $1 \cdot 13(6)$ & $0.3778(6)$ & $3 \cdot 3$ & $7 \cdot 2$ \\
\hline Albasiny \& Cooper (1963) & $16.87(26)$ & $1 \cdot 12(4)$ & $1 \cdot 14(4)$ & $0.3778(4)$ & $2 \cdot 8$ & $4 \cdot 6$ \\
\hline Isolated atoms & & & & & & \\
\hline With N-H $d=1.95$ & $16.96(30)$ & $1 \cdot 16(4)$ & $1 \cdot 11(6)$ & $0 \cdot 3781(4)$ & $2 \cdot 5$ & $5 \cdot 3$ \\
\hline $1 \cdot 75$ & $16.95(22)$ & $1 \cdot 15(4)$ & $1 \cdot 11(4)$ & $0.3780(4)$ & $2 \cdot 0$ & $4 \cdot 0$ \\
\hline
\end{tabular}

Table 3. Results of the 2 parameter (overall scale factor, overall isotropic temperature factor in addition to individual input values) refinement $\mathrm{NH}_{4} \mathrm{~F}$ crystal structure employing neutron diffraction information

\begin{tabular}{|c|c|c|c|c|c|}
\hline \multirow[b]{2}{*}{ Wave function employed for $\mathrm{NH}_{4}{ }^{+}$} & \multicolumn{3}{|c|}{ Refined parameters } & \multicolumn{2}{|c|}{ Agreement } \\
\hline & o.s.f. & $B_{F}$ & $\left(B_{\mathrm{N}}\right)$ & $R$ & $\boldsymbol{R}_{w}$ \\
\hline Krauss (1963) & $16 \cdot 47(16)^{*}$ & $1 \cdot 07(2)$ & $1 \cdot 11$ & $1.9 \dagger$ & $3.0 \dagger$ \\
\hline Moccia (1964) & $16.94(40)$ & $1 \cdot 11(6)$ & $1 \cdot 15$ & $3 \cdot 1$ & $7 \cdot 3$ \\
\hline Albasiny \& Cooper (1963) & $16 \cdot 82(26)$ & $1 \cdot 10(4)$ & $1 \cdot 14$ & $2 \cdot 7$ & $4 \cdot 6$ \\
\hline Isolated atoms with $\mathrm{N}-\mathrm{H} d=1.95$ & $16 \cdot 84(30)$ & $1 \cdot 12(4)$ & $1 \cdot 16$ & $2 \cdot 5$ & $5 \cdot 5$ \\
\hline $1 \cdot 75$ & $16 \cdot 83(24)$ & $1 \cdot 11(4)$ & $1 \cdot 15$ & 1.9 & $4 \cdot 3$ \\
\hline
\end{tabular}


The evidence listed supports the choice of $\mathrm{NH}_{4} \mathrm{~F}$ as a test case for application of the $\mathrm{NH}_{4}^{+}$molecular ion form factor.

For comparison of calculated predictions with experiment, we use Adrian's $\mathrm{NH}_{4} \mathrm{~F}$ single-crystal X-ray diffraction data, obtained with Mo radiation at $-155^{\circ} \mathrm{C}$ (175 reflexions, 95 of them independent). These define the sets $\left\{I_{\mathrm{obs}}\right\}$ and $\{\mathbf{s}(a, b, c)\}$ mentioned in Fig. 1. The $\mathrm{NH}_{4} \mathrm{~F}$ structure will here be resolved in terms of distinct $\mathrm{NH}_{4}^{+}$and $\mathrm{F}^{-}$ions. There are two different orientations for the $\mathrm{NH}_{4}^{+}$ion (outcome of analysis (1) in terms of atoms) defining the set [ $\left.\mathbf{K}_{j}\right]$ while for the spherically symmetric $\mathrm{F}^{-}$ion no transformation of $\{\mathbf{s}\}$ is necessary. The sets $\left[\left\{\mathbf{s}\left(x_{j}, y_{j}, z_{j}\right)\right\}\right]$ are thus defined and the corresponding sets $\left[\left\{f_{j}\right\}\right]$ were calculated for all wave functions of Table 1 . For the $\mathrm{F}^{-}$ion only one wave function was employed (Clementi, 1965).

A set of structure factors $\left\{F_{\text {calc }}(\mathbf{s})\right\}$ may now be calculated according to equation (8) for each wave function of Table 1 and compared with the observed intensities $\left\{I_{\mathrm{obs}}\right\}$. Remaining parameters (overall scale factor, molecular positions and temperature factors) may be adjusted with a suitable refinement procedure. For this purpose, the $O R F L S$ least-squares refinement program (Busing, Martin \& Levy, 1962) was modified (Adrian, 1968) to read the elements of the sets $\left\{f_{j}\right\}$ as input data, instead of employing regular atomic scattering factor tables.

Various refinements (different numbers of adjustable parameters; different weighting schemes) were performed and the results presented in Tables 2 and 3 are representative. The isolated-atoms wave function was employed with several $\mathrm{N}-\mathrm{H}$ distances. Two of these are given in the Tables, corresponding to the distances determined by neutron and X-ray diffraction. The $R$ values listed are defined as usual in X-ray crystallography:

$$
\begin{aligned}
& R=\Sigma \Delta F / \Sigma\left|F_{\mathrm{obs}}\right|, \\
& R_{w}=\left\{\Sigma(w \Delta F)^{2} / \Sigma\left(w\left|F_{\mathrm{obs}}\right|\right)^{2}\right\}^{1 / 2},
\end{aligned}
$$

where the summations are over all observed reflexions and $\Delta F=|| F_{\text {obs }}|-| F_{\text {calc }} \|$. The weighting scheme employed for the refinements of Tables 2 and 3 is $w^{-1}=$ $\left|F_{\text {obs }}\right|$ for all reflexions. For the 2 parameter refinement of Table 3 the maximum amount of information from neutron diffraction structure analysis has been used: the positional parameter, $u,(0 \cdot 3780)$ and individual isotropic temperature factors $\left(B_{\mathrm{F}}=0.82\right.$ and $B_{\mathrm{N}}=$ 0.86 at $-196^{\circ} \mathrm{C}$ ). To make up for the temperature difference between the two experiments, a common increment was added to these $B$ values in the refinement.

It will be seen that analysis employing the molecular form factor calculated from Krauss's multi-centre wave function comes out best, judged by the values of the $R$ values. The one-centre functions, however, though of appreciably lower energy than the isolated-atoms function, compare less favourably with the latter in predicting X-ray scattering, especially when the $\mathrm{N}-\mathrm{H}$ distance of the isolated atoms is decreased (which corresponds actually to refinement of an additional parameter). It is, therefore, concluded that the reliability of molecular form factors is mostly determined by the presence of the appropriate number of centres in the wave function basis, and only then by the predicted energy of the system.

There is reason to suppose that in the comparison discussed the discrepancies between the different wave functions, and hence between the corresponding form factors, have partly been compensated for by the adjustable parameters (note the differences in the refined overall scale factors). This may be judged from the following analysis.

In Table 4, X-ray scattering as predicted by the various wave functions is compared on a purely theoretical basis. For this purpose a fictitious $\mathrm{NH}_{4}^{+}$crystal structure is created by reducing the scattering power of the $\mathrm{F}^{-}$ion in $\mathrm{NH}_{4} \mathrm{~F}$ to zero. Pairs $(i, j)$ of sets $\left\{F_{\text {calc }}\right\}$ obtained for different wave functions $(i, j)$ may now be compared (without any adjustable parameters) and pairwise disagreement, as indicated by the $\mathrm{R}(i, j)$ values, evaluated. The set $\{\mathbf{s}\}$ employed is the same as the one used before. The $R$ values are defined as in equations (9) and (10) with $F_{\text {obs }}=F_{\text {calc }}(i)$ and $F_{\text {calc }}=F_{\text {calc }}(j)$. The differences here are more pronounced, suggesting the compensating effect of the refined parameters in the $\mathrm{NH}_{4} \mathrm{~F}$ analysis.

\section{Conclusion}

The calculation of molecular form factors and their application in X-ray crystallography has been discussed. Within the present approach the electron density in crystalline solids is resolved in terms of molecules instead of atoms. Intermolecular chemical binding is

Table 4. Comparison of $\mathrm{NH}_{4}^{+} X$-ray scattering as predicted by different wave functions

$\begin{array}{lccccc}\text { Wave function } & \text { Krauss } & \text { Moccia } & \text { Albasiny } & \begin{array}{c}\text { Isolated } \\ \text { atoms }\end{array} & \begin{array}{c}\text { Isolated } \\ \text { atoms }\end{array} \\ \text { N-H distance } & 1 \cdot 9464 & 1 \cdot 99 & 1 \cdot 90 & 1 \cdot 95 & 1 \cdot 75 \\ \text { Krauss (1963) } & - & 3 \cdot 6 * & 3 \cdot 0 & 4 \cdot 2 & 3 \cdot 1 \\ \text { Moccia (1964) } & 8 \cdot 7 \dagger & - & 1 \cdot 9 & 5 \cdot 9 & 4 \cdot 6 \\ \text { Albasiny \& Cooper (1963) } & 6 \cdot 3 & 5 \cdot 3 & - & 5 \cdot 6 & 4 \cdot 3 \\ \text { IA (1.95) } & 6 \cdot 9 & 8 \cdot 2 & 7 \cdot 2 & - & 1 \cdot 7 \\ \text { IA (1.75) } & 5 \cdot 2 & 7 \cdot 8 & 5 \cdot 2 & 2 \cdot 9 & -\end{array}$

* Upper right entries: $R$ in \%.

$\dagger$ Lower left entries: $R_{w}$ in \%. 
properly accounted for, but the molecules are treated as rigid scatterers, favouring application at lower temperatures. The method has been applied to the $\mathrm{NH}_{4}^{+}$ molecular ion in $\mathrm{NH}_{4} \mathrm{~F}$. The results of this analysis show that within the rigid scatterer approximation the model employing a molecular form factor for $\mathrm{NH}_{4}^{+}$ calculated from a multi-centre wave function agrees better with experiment than the isolated-atoms model (even with the $\mathrm{H}$ atoms optimally displaced inwards the bond directions in order to compensate for the effect of chemical binding). Molecular form factors derived from one-centre wave functions are less satisfactory; apparently their shortcomings in reproducing the one-electron probability density near the off-centres outweigh their ability to account for chemical binding. In this connexion the results of the analysis of electron diffraction data on methane with a one-centre wave function (Iijima, 1965) are surprising.

The test case chosen is probably not the most profitable one, as the effect of chemical binding on a $\mathrm{H}$ atom may quite well be represented by displacement (and contraction) of its electron density (Stewart, Davidson \& Simpson, 1965). As another case for application, the scattering of the $\mathrm{CN}^{-}$ion is currently being studied. In general, the limitations for application of molecular form factors are determined by the availibility of $a b$ initio molecular wave functions, implying a restriction to structures that may be resolved in terms of small molecules or molecular ions.

\section{APPENDIX}

The problem of calculating molecular form factors from molecular wave functions requires evaluation of elements $f_{p q}(\mathbf{s}), c f$. equation (6), which are given here in the form:

$$
\begin{gathered}
f_{p q}(\mathbf{s})=\int d \mathbf{r} \chi_{p}\left(\mathbf{r}-\mathbf{R}_{p}\right) \chi_{q}\left(\mathbf{r}-\mathbf{R}_{q}\right) \exp (i \text { s. } \mathbf{r}) \\
=\exp \left(i \mathbf{s} . \mathbf{R}_{p}\right) \int d \mathbf{r} \chi_{p}(\mathbf{r}) \chi_{q}\left(\mathbf{r}+\mathbf{R}_{p q}\right) \exp (i \mathbf{s} . \mathbf{r}),
\end{gathered}
$$

where $\chi_{p}$ and $\chi_{q}$ refer to their centres of factorization. For OC integrals $\mathbf{R}_{p q}=\mathbf{R}_{p}-\mathbf{R}_{q}=0$.

For a Gaussian basis $\left\{\chi_{p}\right\}$ both OC and TC integrals can be evaluated analytically, if the functions $\chi_{p}$ are of the form

$$
\begin{aligned}
\chi_{p}(\mathbf{r}) & =r^{2 n_{p}+l_{p}} \exp \left(-\alpha_{p} r^{2}\right) S_{l_{p} m_{p}}(\theta, \varphi) \\
& =\varrho_{p}(r) P_{l_{p} m_{p}}(x, y, z),
\end{aligned}
$$

where $P_{l m}$ is a polynomial of degree $l$ in $x=r \sin \theta \cos \varphi$, $y=r \sin \theta \sin \varphi$ and $z=r \cos \theta$, corresponding to $r^{l} S_{l m}$, and $\varrho_{p}(r)$ is defined here slightly different from equation (7) and must be even in $r$. Different terms in $P$ are treated individually and in the following we understand $P_{l}$ to be a single product in $x, y$ and $z$ of degree $l$. The reduction of $(6 a)$ is then as follows.

$$
\begin{aligned}
& f_{p q}(\mathbf{s})=\exp \left(i \mathbf{s} . \mathbf{R}_{p}\right) \int d \mathbf{r} \varrho_{p}(r) P_{l_{p}}(x, y, z) \chi_{q}\left(\mathbf{r}+\mathbf{R}_{p q}\right) \\
& \times \exp (i \mathbf{s} \cdot \mathbf{r})
\end{aligned}
$$

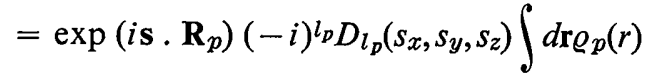

$$
\begin{aligned}
& \times \chi_{q}\left(\mathbf{r}+\mathbf{R}_{p q}\right) \exp (i \mathbf{s} . \mathbf{r}),
\end{aligned}
$$

where $D_{l}$ denotes an operator of differentiation with respect to $s_{x}, s_{y}, s_{z}$. Application of $D_{l}$ to $\exp (i \mathbf{s} . \mathbf{r})$ produces the function $P_{l}(x, y, z) i^{l_{p}} \exp (i \mathbf{s} . \mathbf{r})$. The remaining integral is further reduced by

$$
\begin{aligned}
\int d \mathbf{r} \varrho_{p}(r) \chi_{q}(\mathbf{r} & \left.+\mathbf{R}_{p q}\right) \exp (i \mathbf{s} . \mathbf{r})=(-1)^{n_{p} D_{n_{p}}\left(\alpha_{p}\right)} \\
& \times \int d \mathbf{r} \varrho_{p}^{0}(r) \chi_{q}\left(\mathbf{r}+\mathbf{R}_{p q}\right) \exp (i \mathbf{s} \cdot \mathbf{r})
\end{aligned}
$$

with $\varrho_{p}^{0}(r)=\exp \left(-\alpha_{p} r^{2}\right)$ and $D_{n}\left(\alpha_{p}\right)$ denoting the $n$th order derivative with respect to $\alpha_{p}$. The integral now remaining is further reduced by first shifting the origin of the coordinate system of integration to the centre of $\chi_{q}$ and applying the same reduction technique. The result is

$$
\begin{aligned}
f_{p q}(s) & =(-i)^{2\left(n_{p}+n_{q}\right)+l_{p}+l_{q}} \\
& \times \exp \left(i \mathbf{s} . \mathbf{R}_{p}\right) D_{l_{p}}\left(s_{x}, s_{y}, s_{z}\right) D_{n_{p}}\left(\alpha_{p}\right) \\
& \times \exp \left(-i \mathbf{s} . \mathbf{R}_{p q}\right) D_{l_{q}}\left(s_{x}, s_{y}, s_{z}\right) D_{n_{q}}\left(\alpha_{q}\right) \\
& \times \int d \mathbf{r} \varrho_{p}^{0}\left(\left|\mathbf{r}-\mathbf{R}_{p q}\right|\right) \varrho_{q}^{0}(r) \exp (i \mathbf{s} . \mathbf{r})
\end{aligned}
$$

where it has been tacitly assumed that the orientations $(\theta, \varphi)$ at the centres $p$ and $q$ defined by (11) are the same. The integral at the end of (14) is easily evaluated and is equal to

$\left(\frac{\pi}{\alpha_{p}+\alpha_{q}}\right)^{3 / 2} \exp \left\{\frac{-4 \alpha_{p} \alpha_{q} R_{p q}^{2}+4 i \alpha_{p} \mathbf{R}_{p q} \cdot \mathbf{s}-s^{2}}{4\left(\alpha_{p}+\alpha_{q}\right)}\right\}$

as already obtained by McWeeny (1953), who also treated some special cases of the above problem though by a somewhat different approach. All elements $f_{p q}(\mathbf{s})$ can thus be obtained by differentiation of simple analytic basic forms (15). For OC integrals the procedure is even more simple, as $\mathbf{R}_{p q}=0$ and all differentiation operations may be permuted to convenience.

For a Slater basis the same technique is applicable for OC integrals. The differentiations $D_{n}$ are now with respect to the Slater exponential coefficients $\zeta$ and the final analytic basic form is

$$
\begin{aligned}
& \int d \mathbf{r} r^{n_{p}+n_{q}-2} \exp \left(-\zeta_{p} r-\zeta_{q} r+i \mathbf{s} . \mathbf{r}\right)=4 \pi \frac{\Gamma\left(n_{p}+n_{q}\right)}{s} \\
& \quad \times\left\{\left(\zeta_{p}+\zeta_{q}\right)^{2}+s^{2}\right\}^{-\left(n_{p}+n_{q}\right) / 2} \\
& \quad \times \sin \left\{\left(n_{p}+n_{q}\right) \operatorname{arctg} \frac{s}{\zeta_{p}+\zeta_{q}}\right\},
\end{aligned}
$$

also obtained by McWeeny (1951). This formula is valid for non integral $n_{p}$ and $n_{q}$ also. For TC integrals with a Slater basis, McWeeny's approach, of projecting the Slater functions on a suitable Gaussian basis, seems 
a feasible method which keeps the advantages of the above reduction procedure (McWeeny, 1953; for different approaches to the evaluation of some TC Slater integrals cf. Bonham, Peacher \& Cox, 1964; Guerillot, Ganachaud \& Lissillour, 1968).

When the final form cannot be evaluated analytically, the method above of replacing integration by differentiation is useless. This is the case when the radial basis $\left\{\varrho_{p}\right\}$ is numerical. However, for OC integrals, the threedimensional integrals over $\mathbf{r}$ can be reduced to onedimensional ones by similar techniques. Here

$$
\begin{aligned}
& f_{p q}(\mathbf{s})=\int_{0}^{\infty} d r r^{2} \varrho_{p}(r) \varrho_{q}(r) \int d \omega P_{l_{p}}(x, y, z) P_{l_{q}}(x, y, z) \\
& \times \exp (i \mathbf{s} \cdot \mathbf{r})=\int_{0}^{\infty} d r r^{2} \varrho_{p}(r) \varrho_{q}(r) \\
& \times 4 \pi(-i)^{l_{p}+l_{q} D_{l_{p}+l_{q}}\left(s_{x}, s_{y}, s_{z}\right) j_{0}(s r),}
\end{aligned}
$$

where $j_{0}$ is the zero order spherical Bessel function. Application of $D_{l}$ to $j_{0}$ is easily performed employing the properties of the functions $j_{n}$. Computation of the remaining integral over $r$ requires evaluation of $j_{n}(s r)$ up to $n=l_{p}+l_{q}$ at all argument values of the numerically defined $\varrho p$. A different treatment of this problem was given by Freeman (1959).

We thank Mr H.W.W.Adrian for many creative discussions and for the ready access he gave us to the results of his investigation.

\section{References}

Adrian, H. W. W. (1968). M. Sc. Thesis, Physics Department, Univ. of Cape Town. Unpublished.

Adrian, H. W. W. \& FeIL, D. (1969). Acta Cryst. 25, 438.

Albasiny, E. L. \& Cooper, J. R. A. (1963). Proc. Phys. Soc. (London), 82, 289.

Bonham, R. A., Peacher, J. L. \& Cox, H. L. JR (1964). J. Chem. Phys. 40, 3083.

Busing, W. R., Martin, K. O. \& Levy, H. A. (1962). ORFLS. Oak Ridge National Laboratory, Oak Ridge, Tennessee.

Clementi, E. (1965). Tables of Atomic Functions. San Jose: I.B.M. Corporation.

Davis, M. F. \& Whitaker, A. (1966). Acta Cryst. 21, 822.

Dawson, B. (1964). Acta Cryst. 17, 997.

Freeman, A. J. (1959). Acta Cryst. 12, 261.

Guerillot, C. R., Ganachaud, J. P. \& Lisslllour, R. (1968). Theor. Chim. Acta 9, 230.

IIJMa, T., BonhaM, R. A., TavaRD, C. \& Roux, M. (1965). Bull. Chem. Soc. Japan, 38, 1757.

James, R. W. (1948). The Optical Principles of the Diffraction of $X$-rays. p. 223. London: Bell.

Krauss, M. (1963). J. Chem. Phys. 38, 564.

Moccia, R. (1964). J. Chem. Phys. 40, 2164.

Stewart, R. F., Davidson, E. R. \& Simpson, W. T. (1965). J. Chem. Phys. 42, 3175.

WebB, M. W. (1965). Acta Cryst. 18, 298.

McWeeny, R. (1951). Acta Cryst. 4, 513.

MCWeEny, R. (1952). Acta Cryst. 5, 463.

MCWeEny, R. (1953). Acta Cryst. 6, 631.

McWeeny, R. (1954). Acta Cryst. 7, 180.

Acta Cryst. (1969). A 25, 450

\title{
The Deformations of the Ions in $\mathrm{NaCl}$ and $\mathrm{AgCl}$ Crystals and the Temperature Parameters of Ions in Some Alkali Halides
}

\author{
BY M.V.LINKOAHO \\ Laboratory of Physics, Technical University of Helsinki, Otaniemi, Finland
}

(Received 16 September 1968)

\begin{abstract}
Results of X-ray powder intensity measurements and spherical harmonic expansions have been used for investigating deformations of the ions in $\mathrm{NaCl}$ and $\mathrm{AgCl}$ crystals. It is found that within the limits of experimental accuracy the ions in crystalline $\mathrm{NaCl}$ are spherically symmetric. Compared with free ions, the charge around $\mathrm{Ag}$ in $\mathrm{AgCl}$ has spread farther and a part of it is located inside the $\mathrm{Cl}$ sphere. The electron clouds inside the $\mathrm{Ag}$ and $\mathrm{Cl}$ spheres are, however, almost spherically symmetric. It has been shown that the results of recent X-ray measurements concerning the Debye-Waller coefficients of the ions in alkali halide crystals have one prominent feature: the square of the relative vibration amplitude of an individual ion, which is proportional to the ratio $B / a^{2}(B$ is the Debye-Waller coefficient and $a$ the length of the edge of the unit cell), increases when the number of electrons of its company increases. It is proposed that this dependence is mainly due to the repulsive forces between the next-nearestneighbours.
\end{abstract}

\section{Introduction}

Iti.has been often proposed that there are some unspherical deformations of the ions of alkali halide crystals in spite of the high symmetry of these crystals and of their ideal ionic character. For example, Korhonen (1956) has analysed the measurements of Wasastjerna (1944) and found deformations in the electron 